\title{
Bridging the Gap between Aspect Mining and Refactoring
}

\author{
Isaac Yuen and Martin P. Robillard \\ School of Computer Science \\ McGill University \\ \{iyuen|martin\}@cs.mcgill.ca
}

\begin{abstract}
Aspect-mining techniques help to identify crosscutting structure that could potentially be modularized through objectoriented $(\mathrm{OO})$ or aspect-oriented refactoring $(\mathrm{AO})$. This paper describes a case study in which we used aspect-mining techniques to identify and refactor crosscutting concerns using aspect-oriented programming. We observed that, in our case, there were many subtle variations in the implementation of the concerns that made them non-trivial to modularize with AO refactoring. In the end, we solved our modularization problem using traditional $\mathrm{OO}$ refactoring. We conclude that there exists an important gap between the identification of crosscutting concerns and the technologies available to mitigate the problem.
\end{abstract}

\section{INTRODUCTION}

Crosscutting concerns in a software system cause many problems, including scattered duplicate code. As a result, the system gradually becomes less maintainable. Aspectoriented programming (AOP) proposes a solution to the crosscutting problem by supporting the modularization of crosscutting concerns into aspects. The availability of aspects suggests that it should be possible to incrementally refactor an existing object-oriented (OO) system into a more modularized AO equivalent. Nevertheless, this strategy poses at least three issues that must be addressed:

1. How to identify crosscutting concerns in a system;

2. How to determine if the concern code identified in step 1 is refactorable;

3. How to refactor crosscutting concerns into aspects, either manually or automatically.

Aspect mining addresses the first problem by identifying potential crosscutting concerns in a system using techniques such as fan-in analysis [15], lexical and dynamic analyses [6], and version history mining [4]. For crosscutting concerns that span many classes in a system, there exist tools that

Permission to make digital or hard copies of all or part of this work for personal or classroom use is granted without fee provided that copies are not made or distributed for profit or commercial advantage and that copies bear this notice and the full citation on the first page. To copy otherwise, to republish, to post on servers or to redistribute to lists, requires prior specific permission and/or a fee.

Workshop LATE '07 March 12-13, 2007 Vancouver, British Columbia, Canada

Copyright 2007 ACM 1-59593-655-4/07/03 ...\$5.00. can help refactor the targeted code into an aspect automatically [2]. Such tools can reduce the effort required and the risks of introducing error typically associated with manual refactoring. However, between the identification of crosscutting concerns and the refactoring process, a developer must determine if the crosscutting concerns are refactorable. In particular, there are several issues that we must consider before applying refactoring, including:

- Does the AOP language support the extraction of the targeted crosscutting code?

- Can we deduce a pattern in the crosscutting code, such that we can apply a general pointcut that captures all relevant join points and produces a better modularized system?

- If we find variants in the crosscutting code that do not conform to the common patterns, what kinds of measure can we take to eliminate the discrepancy?

We conducted a case study to explore these questions by evaluating some crosscutting concerns in an open-source project. We employed fan-in analysis [15] as the aspect mining tool to help us locate the crosscutting concerns in the target system. We then manually inspected the results of the aspect-mining technique, and determined if there was an applicable AO refactoring [17] that could help us extract these crosscutting concerns into an aspect. We concluded that due to the variations in the code, it was difficult to choose a refactoring that neatly encapsulated the crosscutting concerns without sacrificing the readability and simplicity of the pointcut descriptor.

We resolved to refactor our crosscutting concerns by incrementally applying traditional OO refactoring such as Extract Method and Pull Up Method [8]. This strategy simplified the code and revealed some previously unseen relations between classes that participate in the implementation of the same crosscutting concern. We argue that comprehensive OO refactoring be a required preliminary step in assessing the applicability of AO refactoring.

\section{CASE STUDY SETUP}

The initial goal of our case study was to inspect crosscutting code and assess the difficulty in refactoring it into aspects. We chose an open-source Java project as our experimental target, and assumed AspectJ as the targeted AOP language for refactoring. 


\subsection{Target Systems}

We applied our case study to an open-source project called Freemind $^{1}$. FreeMind is a mind-mapping software written in Java. It is an editor for creating and navigating hierarchical diagrams. The version we used (0.8.0) consists of approximately 65,600 non-comment lines of code. FreeMind has over 5 years of development history and is currently maintained by 4- 6 developers. FreeMind's medium user base ( $\sim 100,000$ users) and its reputation ${ }^{2}$ convince us that it is a representative example of a medium size Java project.

\subsection{Locating crosscutting candidates}

Fan-in analysis is an aspect-mining technique that identifies crosscutting concerns whose implementation consists of a large number of scattered invocations of specific functionality implemented by a method. The number of distinct calling methods gives the fan-in metric of the invoked method [14]. The FINT ${ }^{3}$ Eclipse plug-in is a tool that supports fan-in analysis and navigation of crosscutting concerns, and was used as the aspect-mining tool in this case study. The results of FINT provided us with the fan-in values for all methods in the system. Since FINT does not provide information about the locations of the crosscutting calls, we manually inspected the call sites of each crosscutting candidate that has a fan-in value of at least 8 to determine if it forms a refactorable crosscutting concern.

\subsection{Criteria for refactorable concerns}

There is no formal guide to determine precisely what a refactorable crosscutting concern consists of. Therefore, we choose to look for a crosscutting concern that satisfies the following criteria:

Occurrence: A refactorable crosscutting concern should crosscut at least 8 different method bodies ${ }^{4}$ in the system. In particular, we looked for clusters of at least 3 method invocations that can be found in all of the crosscutted method bodies.

Pattern: There should be a consistent pattern with respects to the order of invocations of these methods.

Absence of complex control structures: AspectJ does not have pointcut descriptors for control-flow such as loop, or switch statements [11]. Complex control structures also introduce irregularities among the crosscutting call sites. Therefore, we only considered crosscutting code that was not nested in control structure.

\section{CASE STUDY RESULTS}

We identified two refactorable crosscutting concerns using fan-in analysis. For each, we describe the nature of the concern's implementation, the challenges it poses to AO refactoring, our proposed solution, and our observations.

\subsection{Overview of crosscutting candidates}

The fan-in analysis of FreeMind reported a number of methods with fan-in higher than 100. However, we found that candidates that have high fan-in values are often not

\footnotetext{
${ }^{1}$ http://freemind.sourceforge.net

${ }^{2}$ FreeMind was chosen as SourgeForge Project of the Month in February 2006.

${ }^{3}$ http://swerl.tudelft.nl/bin/view/AMR/FINT (v0.6)

${ }^{4}$ The default threshold fan-in value of FINT is 8
}

refactorable, either because they reside inside some controlflow structure (such as a switch statement), or because the locations of the call sites do not form any pattern. However, we located two groups of methods, with fan-in values of 32 and 49 respectively, that exhibit some recurring code patterns for refactoring. We outline the evaluation of each concern in the following sections.

\subsection{Action Concern}

Common pattern in crosscutting code. From the results of fan-in analysis, we noticed a group of methods that are invoked in the same pattern consistently, and whose fan-in value is equal or close to the other methods in the group. We identified that this group contains a code clone, and this clone is found in 32 different method bodies (see Table 1).

Figure 1 shows an example of this clone in AddArrowLink Action. java. In general, the clone consists of three method calls that are always in the same order: a call to executeTransaction() is preceded by a call to startTransaction() and followed by a call to endTransaction(). In most cases, their call sites are adjacent to each other inside a method body. The fact that the clones are not located inside a loop or a branch makes the sequence of calls a good target for refactoring.

\begin{tabular}{|l|c|}
\hline Crosscutting calls & Fan-in \\
\hline ActionFactory.startTransaction(String)) & 33 \\
\hline ActionFactory.executeAction(ActionPair) & 37 \\
\hline ActionFactory.endTransaction(String) & 33 \\
\hline \hline Intersection & 32 \\
\hline
\end{tabular}

Table 1: Fan-in values for methods of the Action Control concern

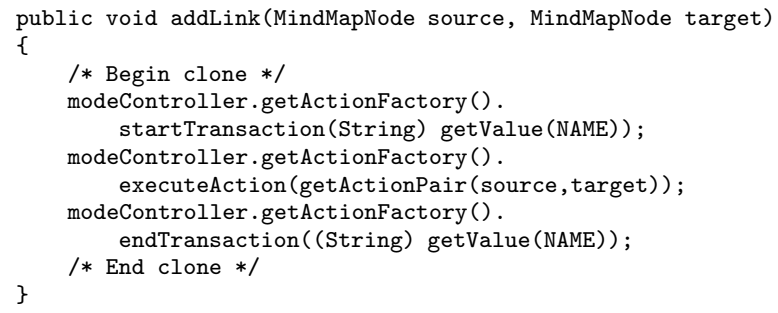

Figure 1: Action Control concerns in AddArrowLinkAction. java

Challenges for $A O$ refactoring. The code for this concern exhibits a consistent behaviour [13], and the method signatures reveal that it acts as a transactional control. There are two options to extract the crosscutting code into an aspect:

Option 1. Refactor the calls to startTransaction() and endTransaction() into an 'around' advice of the containing method.

This options requires that startTransaction() and endTransaction() always be located at the beginning and end of the method body, which is not true for all cases. Figure 2 shows a variant that contains some initialization code before the clone.

Option 2. Refactor the startTransaction() and endTransaction() into an 'around' advice of executeAction(). While the approach solves the problem created by the variant in 


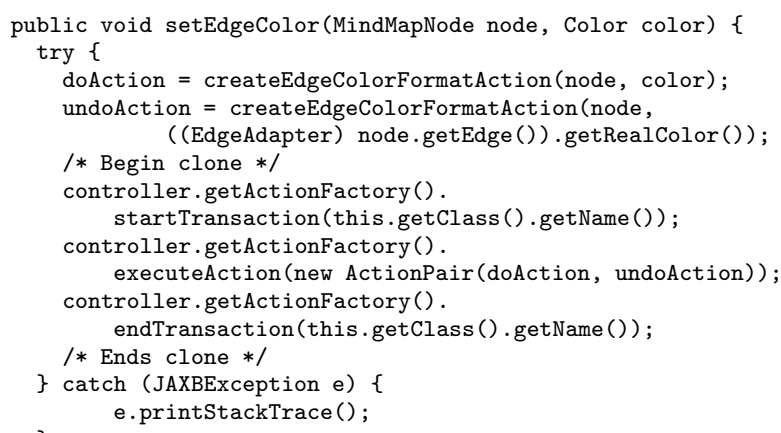

Figure 2, it creates two new challenges. First, there are also variants of the clone in which executeAction() is not called right after the startTransaction() (see Figure 3). The fanin analysis (Table 1) also shows that executeAction() has a higher fan-in value than the other two methods, and we noticed that there are several exceptions where startTransaction() and endTransaction() are not called along with executeAction(). A pointcut descriptor that accounts for these exceptions would be difficult to create.

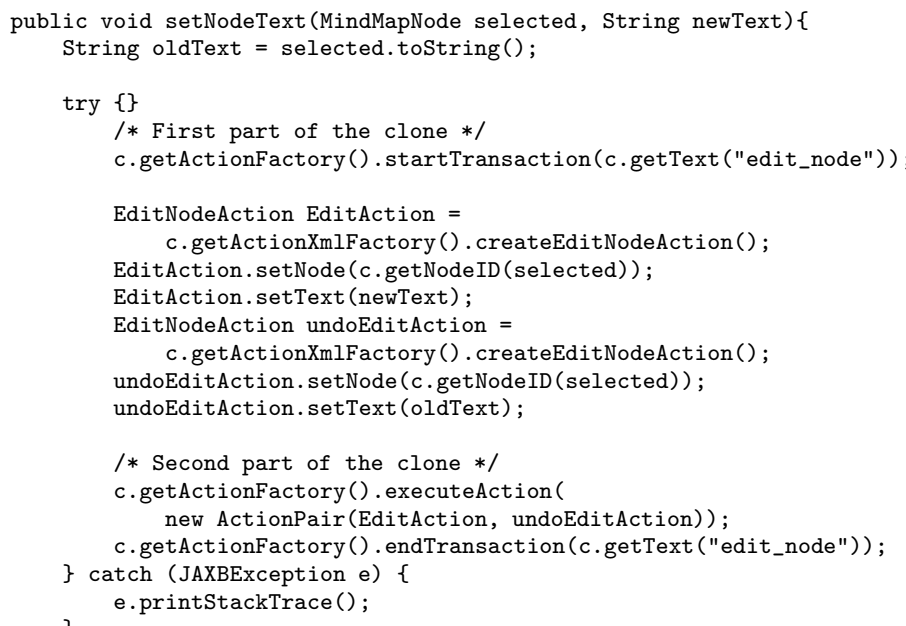

Figure 3: Clone variant in EditAction.java

Refactoring solution. The first step of refactoring must resolve the inconsistency found in Figures 2 and 3. Since in most cases all three calls in the clone are adjacent to each other, the best approach is to 'correct' the type of variant found in Figure 3 and change the locations of startTransaction() and endTransaction() to make them adjacent to the executeAction() call. The main challenge of the change is that we must verify that it does not change the behavior of the containing method body. In all cases of this crosscutting concern, we manually verified that this change was safe.

We then used the Extract Method technique to extract the clone in each class into a new method called runTransaction (see Figure 4).

After the extraction, we discovered an interesting core-

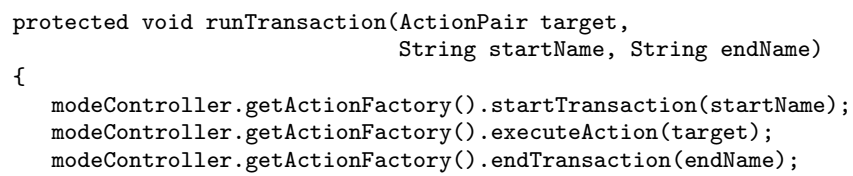

Figure 4: Extracted method runTransaction()

lation between the refactored classes. For instance, most of the classes that contain the clone are descendants of the FreemindAction class, even though the FreemindAction class does not contain the clone. Therefore we applied the Extract Superclass method repeatedly to extract the runTransaction() method into FreemindAction. This refactoring reduced the lines of code count by approximately 300 .

\subsection{XML Attribute Serialization concern}

Common pattern in crosscutting code. From the results of the fan-in analysis, we noticed another group of method calls that represents another refactorable code clone. This group of code clones spans over 49 different method bodies (see Table 2). Figure 5 shows an example of this clone in the AddIconActionTypeImpl.serializeAttributes() method. In fact, every clone exclusively resides in the body of a serializeAttributes() method, and serializeAttributes() implements a method of the XMLSerializable interface. This implies that every class that is involved in this concern implements XMLSerializable. From this information we deduced that the clones belong to the XML Attribute Serialization concern.

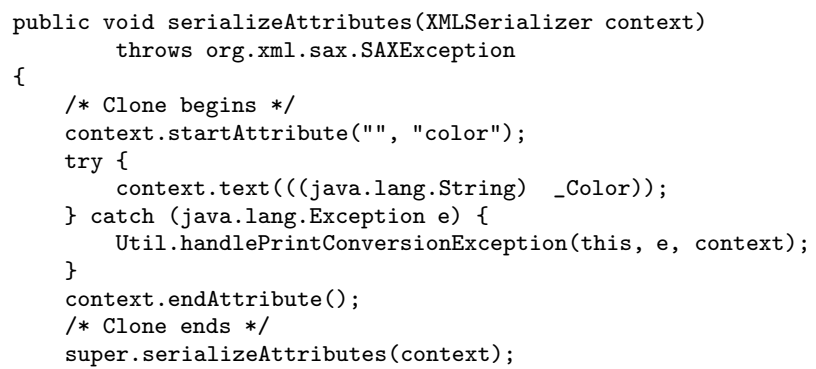

Figure 5: An Example of the refactorable code clone in AddIconActionTypeImpl.java

Challenges with AO refactoring. The XML Attribute Serialization concern consists of an interface implementation and we considered it as a type of role superimposition concern [13]. There are two options to extract the crosscutting clones into an aspect:

Option 1. Refactor the clone into a 'before' advice of serializeAttributes(). Ideally, this option produces the most simplified and modularized implementation of the concern. However, there are variants of serializeAttributes () where this approach is not applicable. For instance, a variant may contain more than one instance of the clone, or the clone may reside in a control structure (e.g. inside an if branch or a while loop) (see Figure 6). Since the variants account for half of the method bodies that contain the clone, we would have to make some exclusions in the pointcut descriptor, and the concern would not be completely 


\begin{tabular}{|l|c|}
\hline Crosscutting calls & Fan-in \\
\hline XMLSerializer.startAttribute(String, String) & 49 \\
\hline XMLSerializer.text(String) & 53 \\
\hline Util.handlePrintConversionException(Object, Exception, XMLSerializer) & 50 \\
\hline XMLSerializer.endAttribute() & 49 \\
\hline \hline Intersection & 49 \\
\hline
\end{tabular}

Table 2: Fan-in values of XML Attribute Serialization concern

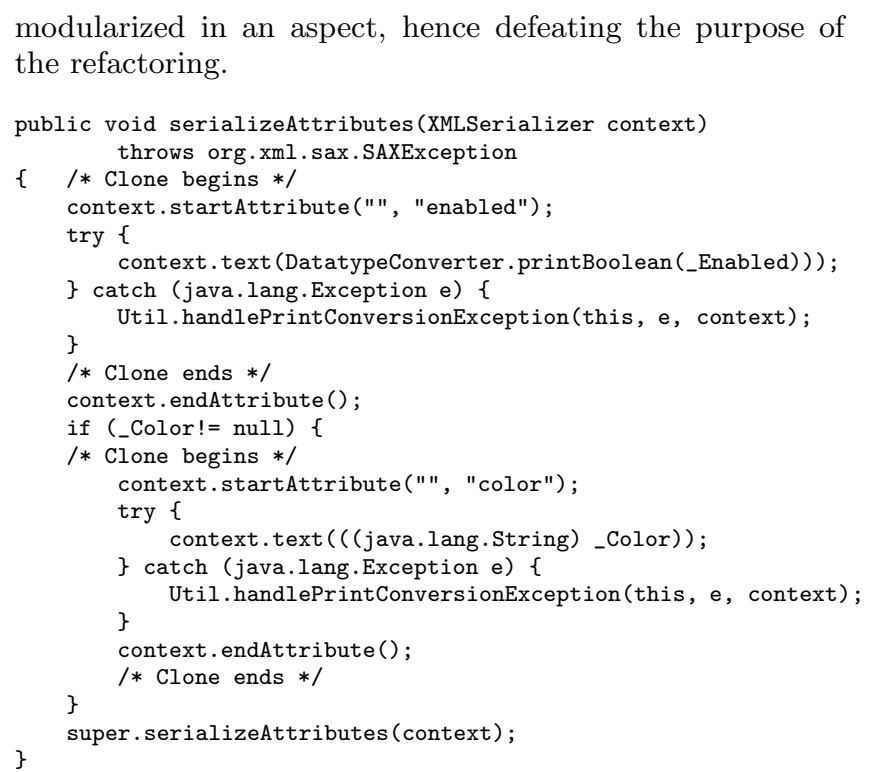

Figure 6: A variant of serializeAttributes() that contains 2 clones.

Option 2. Refactor the clone into an 'around' advice of XMLSerializer.text(). Although this option can be generalized to every instance of the crosscutting call sites, the fanin analysis (Table 2) shows that there are several situations in which XMLSerializer.text() is not called along with XMLSerializer .startAttribute() and XMLSerializer.endAttribute(). We must account for these exceptions in the pointcut descriptor.

Again, due to the irregularity of the crosscutting clones, neither option can provide a refactoring that completely and neatly encapsulates the crosscutting concern into an aspect. However, we could apply the same object-oriented refactoring strategy as the previous concern to eliminate the clones.

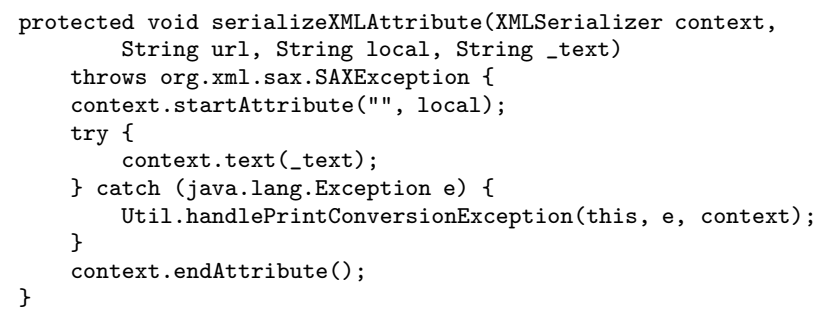

Figure 7: Extracted method serializeXMLAttribute() from the crosscutting code clones

Refactoring solution. . We applied the Extract Method technique to extract the clone into a new method called serializeXMLAttribue() (see Figure 7):
When we inspected the inheritance relationship between the classes that contain the crosscutting clone, we noticed that 33 of the 49 classes declaring our extracted method are descendants of the XmlActionImpl class. Therefore, we repeatedly applied the Pull Up Method to serializeXMLAttribute() until we reached the XmlActionImpl class. The refactored version reduced the line of code count by approximately 200 lines.

Using the inheritance graph, we also noticed that there are several classes that share the same prefix, such as MenuAction BaseImpl, MenuCategoryBaseImpl, and MenuSubmenuTypeImpl. Although these classes do not inherit from any other project class, we deduced that they are conceptually related and should be connected together through a superclass. We applied the Extract Superclass refactoring to refactor the serializeXMLAttribue() method in each class to a new superclass AbstractMenuBaseTypeImpl, and further eliminated approximately 90 lines of code.

\section{DISCUSSION}

The road from aspect-mining to aspect refactoring can be long and winding. Although aspect-mining techniques such as fan-in analysis were able to detect crosscutting functionality in our case study, only a small subset of the identified concerns could be refactored into aspects. One reason is that AOP languages such as AspectJ can only capture a small set of program execution points. Furthermore, AO transformations have many restrictions with respects to the structure and pattern of the crosscutting code, and can only be applied directly in certain circumstances [10]. This requirement of uniformity can rarely be expected from a large system, especially open source projects that have evolved over a long period of time. Developers may introduce changes to the code that disrupt the original structure or pattern and that breaks the regularity required for $\mathrm{AO}$ refactoring.

AO refactoring requires an initial stage of $O O$ refactoring. In our case, crosscutting concerns were associated with code clones. There were several benefits to applying traditional OO transformations to refactor these clones. First, by extracting the code clones into a separate method, the intent of the clone becomes explicit, and the total code size is reduced. Secondly, for clone variants that cannot be directly refactored, we can study the discrepancies, which helps us to spot potential bugs in the system. Thirdly, we may be able to reveal some unseen relationships among the classes that share the same clone, and further refactor the code. Finally, untangling a concern is a major challenge in largescale AO refactoring [2]. By repeatedly refactoring the code to a more concise form, our OO refactoring strategy may help us overcome this challenge. 


\section{RELATED WORKS}

Aspect-mining techniques identify the crosscutting concerns in a system either statically or dynamically. Fan-in analysis [15] is a static aspect mining technique that determines the scatteredness of code by identifying methods that are called in many places in the project. Lexical-based analysis derives crosscutting concerns by grouping program elements based on their lexical representations [6]. A versionhistory based approach by Breu and Zimmermann [4] analyzes the addition and evolution of program elements and correlates them with the author and timestamp data from the version control history. This approach is more scalable to large projects since the precision of the crosscutting concerns increases with the project size and history. A case study by Bruntink et al. [5] applies several clone detection techniques to an industrial $\mathrm{C}$ application and analyzes their effectiveness in finding the crosscutting concerns. Dynamic aspect mining [3] depends on run-time program behavior to identify recurring execution patterns that can be classified as a concern. Tonella and Ceccato apply concept analysis [18] to analyze how execution traces are related to class methods and identify related methods as a crosscutting concern.

There are several case studies that evaluates the applicability of AO refactoring. Monteiro et al. [16] and Hannemann et al. [9] separately conducted studies that applied AO refactorings incrementally on small code examples that use design patterns such as the Observer Pattern. Marin also illustrated the AO refactoring approach on JHotDraw project and completely extracted the Undo concern into an aspect [12]. However, the crosscutting concerns identified in these projects do not exhibit the irregularities in our FreeMind examples. Coyler et al. [7] also conducted a case study that seperated the EJB support from an large-scale application server using $\mathrm{AO}$ refactoring.

Automated AO refactoring remains a challenge. Binkley at al. [1] [2] created a semi-automated approach to refactor object-oriented program elements into aspects. Currently, the prototype lacks syntactic checks and oftens provide erronous refactored code and unreadable pointcuts that can be improved. Hannemann et al. [10] developed another semiautomated approach called role-based refactoring. However, the target code must conform to certain design patterns to enable the refactoring and is not flexible for general use.

\section{CONCLUSION}

We described our efforts to locate refactorable crosscutting concerns in an open-source project using an aspectmining technique, and to refactor the identified code to mitigate their crosscutting nature. The concerns that could be refactored were rare, and we discovered that we could not find an appropriate AO refactoring approach that could produce a simpler and more modularized code, due to the variations in the structure. We resorted to a traditional OO refactoring strategy, and discovered some hidden relationships between classes that helped us further simplify the code as a step that could facilitate future AO migration. We conclude that there exists an important gap between the identification of crosscutting concerns and the technologies available to mitigate the problem.

\section{ACKNOWLEDGEMENT}

This work was supported by the Fond Québécois de la recherche sur la nature et les technologies (FQRNT).

\section{REFERENCES}

[1] D. Binkley, M. Ceccato, M. Harman, F. Ricca, and P. Tonella. Automated refactoring of object oriented code into aspects. In Proceedings of the 21st IEEE International Conference on Software Maintenance, pages 27-36, 2005.

[2] D. Binkley, M. Ceccato, M. Harman, F. Ricca, and P. Tonella. Tool-supported refactoring of existing object-oriented code into aspects. IEEE Transactions on Software Engineering, 32(9):698-717, 2006.

[3] S. Breu and J. Krinke. Aspect mining using event traces. In Proceedings of the 19th IEEE international conference on Automated software engineering, pages 310-315, 2004.

[4] S. Breu and T. Zimmermann. Mining aspects from version history. In Proceedings of the 21th IEEE international conference on Automated software engineering, pages 221-230, 2006.

[5] M. Bruntink, A. van Deursen, T. Tourwe, and R. van Engelen. An evaluation of clone detection techniques for crosscutting concerns. In Proceedings of the 20th IEEE International Conference on Software Maintenance, pages 200-209, 2004.

[6] M. Ceccato, M. Marin, K. Mens, L. Moonen, P. Tonella, and T. Tourwe. A qualitative comparison of three aspect mining techniques. In Proceedings on the 13th International Workshop on Program Comprehension, pages 13-22, 2005.

[7] A. Colyer and A. Clement. Large-scale aosd for middleware. In Proceedings of the 3rd international conference on Aspect-oriented software development, pages 56-65, 2004.

[8] M. Fowler, K. Beck, J. Brant, W. Opdyke, and D. Roberts. Refactoring: Improving the Design of Existing Code. Addison-Wesley Professional, June 1999.

[9] J. Hannemann and G. Kiczales. Design pattern implementation in java and aspectj. In Proceedings of the 17th ACM SIGPLAN conference on

Object-oriented programming, systems, languages, and applications, pages 161-173, 2002.

[10] J. Hannemann, G. C. Murphy, and G. Kiczales. Role-based refactoring of crosscutting concerns. In AOSD '05: Proceedings of the 4th international conference on Aspect-oriented software development, pages 135-146, 2005.

[11] E. Hilsdale and J. Hugunin. Advice weaving in AspectJ. In Proceedings of the 3rd international conference on Aspect-oriented software development, pages 26-35, 2004.

[12] M. Marin. Refactoring JHotDraw's Undo concern to AspectJ. In Proceedings of the First Workshop on Aspect Reverse Engineering (WARE)., 2004.

[13] M. Marin, L. Moonen, and A. van Deursen. A classification of crosscutting concerns. In Proceedings of the 21st IEEE International Conference on Software Maintenance, pages 673-676, 2005.

[14] M. Marin, L. Moonen, and A. van Deursen. A common framework for aspect mining based on crosscutting concern sorts. In Proceedings of the 13th 
IEEE Working Conference on Reverse Engineering, pages 29-38, 2006.

[15] M. Marin, A. van Deursen, and L. Moonen. Identifying aspects using fan-in analysis. In Proceedings of 11th Working Conference on Reverse Engineering, pages 132-141, 2004.

[16] M. P. Monteiro and J. M. Fernandes. Refactoring a java code base to aspectj: An illustrative example. In Proceedings of the 21st IEEE International Conference on Software Maintenance, pages 17-26, 2005.

[17] M. P. Monteiro and J. M. Fernandes. Towards a catalog of aspect-oriented refactorings. In Proceedings of the 4 th international conference on Aspect-oriented software development, pages 111-122, 2005.

[18] P. Tonella and M. Ceccato. Aspect mining through the formal concept analysis of execution traces. In Proceedings of the 11th IEEE Working Conference on Reverse Engineering, pages 112-121, 2004. 